\title{
Thin SOI NEMS accelerometers compatible with In-IC integration
}

Eric Ollier, L. Duraffourg, M. Delaye, S. Deneuville, V. Nguyen, P. Andreucci, H. Grange, P. Robert

CEA-LETI

Grenoble, France

R. Dianoux

ESRF

Grenoble, France

\author{
F. Marchi \\ CNRS/LEPES \\ Grenoble, France \\ Thomas Baron \\ STMicroelectronics \\ Crolles, France
}

\begin{abstract}
The paper presents Thin SOI NEMS structures for accelerometers based on Thin SOI technology and compatible with "In-IC" integration. The goal of this work is to demonstrate the feasibility in terms of concept and technological manufacturing. Modeling of Casimir force, development of hybrid e-beam/DUV lithography and FHvapor release, specific AFM characterizations have allowed to design, fabricate and characterize first devices.
\end{abstract}

\section{INTRODUCTION}

NEMS (Nano-Electro-Mechanical-Systems) devices are emerging as a new field with a great deal of interest because they offer in the same time very interesting specific advantages and also compatibility with "In-IC" integration. They can be generally defined as devices collectively fabricated by micro-technologies and including at least one movable mechanical part with dimension $<100 \mathrm{~nm}$.

NEMS offer key attributes such as ultra miniature size elements, low power consumption, potential high resonant frequencies and quality factors, fast response time and also high sensitivity to applied force, external damping or additional mass. As a consequence, NEMS are very promising for ultimate sensing, autonomous sensors, battery-operated systems and can allow new architectures.

Nowadays, NEMS are investigated in order to face the challenges of nano-world and have a better understanding of the fundamental issues of mechanics in the nano-scale [1]. But thanks to the combination of powerful attributes such as unprecedented mass sensitivity and high operational resonance, NEMS opens up the possibility to prospect ultimate inertial mass sensing [2]. Resonant NEMS can also be combined with nanoelectronic [3] to fabricate displacement detectors based on RF-SET (Radio-Frequency Single-Electron Transistor).

NEMS-IC integration is highly suitable since it can enable to decrease the cost of MEMS. In addition, good performances can be expected because the proximity between NEMS and IC enables to suppress a lot of parasitic effects. In particular, "In-IC" integration is very attractive because of its capability to decrease cost, to facilitate packaging thanks to O-level packaging solutions and also to avoid limitations of "above IC" approach. Finally, thin SOI technology is a very good candidate since the same thin layer of single-crystal silicon can be used for both mechanical structures and advanced ICs.

This approach can be attractive for low cost accelerometers for applications such as : hard-drive protection, motion interface for cell phones, handheld PDAs, game controllers and also gesture recognition or motion capture for medical, wellness or sports activities [4].

\section{MODELIND AND DESIGN}

In order to address this kind of applications and demonstrate the feasibility of thin SOI NEMS accelerometers, $50 \mathrm{G}$ and $10 \mathrm{G}$ accelerometers have been designed.

Compatibility with $0.13 \mu \mathrm{m}$ CMOS SOI technology means that the thickness of the Si top layer is $160 \mathrm{~nm}$, instead of a few $10 \mu \mathrm{m}$ in MEMS devices. Two configurations have been designed : In-Plane (IP) accelerometers with sensitive axis included in the plane of the wafer, and Out-of-Plane (OOP) accelerometers with sensitive axis perpendicular to the wafer (Fig.1). An acceleration causes the motion of a proofmass and the corresponding displacement is measured by means of a variation of capacitance between two sets of electrodes. The electrodes are either interdigitated combs (IP) or two parallel plates (OOP).

In order to avoid parasitic deformations and undesirable vibration modes, a rigid mass was designed with typical dimensions of $75 \times 150 \mu \mathrm{m}$. In addition, for IP accelerometers, low cross-sensitivity implies to increase the ratio thickness/width of the mechanical springs, leading to 
$50 \mathrm{~nm}$ wide mechanical springs. In the same time, increase electrical sensitivity means decrease electrostatic gap down to $100-400 \mathrm{~nm}$. Finally, depending on the FS acceleration range and the resolution expected, the length of mechanical springs and the number of teeth of the electrostatic comb have been adapted. Typically, the length of springs and electrostatic teeth range from 1 to $10 \mu \mathrm{m}$.

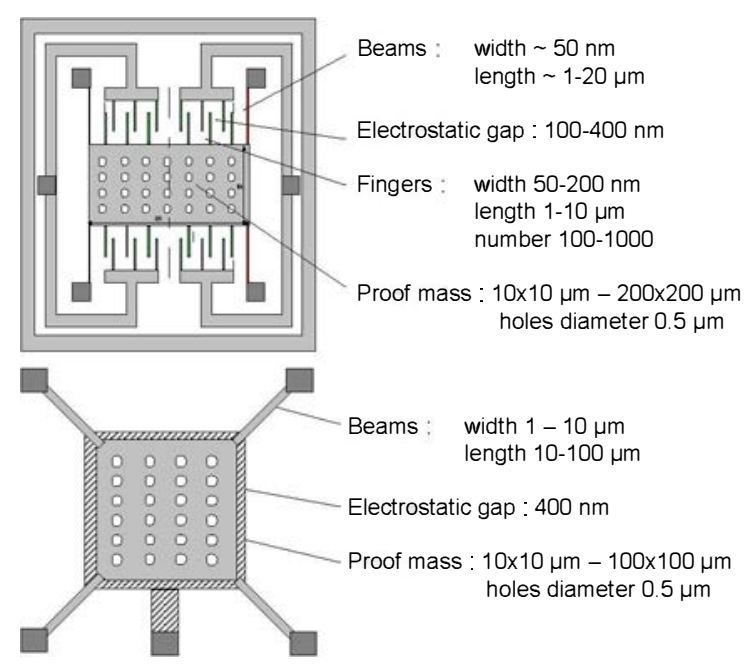

Figure 1. Principle and typical dimensions of IP/ $૦ 2$ accelerometers

Because of thin SOI technology, some dimensions have been reduced drastically compared to traditional thick SOI technology and are now situated in the nano-scale, in particular the mechanical spring width $(50 \mathrm{~nm})$ and the electrostatic gaps (100-400nm). As a consequence, the role of specific phenomenon occurring in the nano-scale has been studied, in particular the role of quantum nongravitational attraction named Casimir force. A new model has been developed for silicon devices in order to take into account doping level and dimensional effects. Fig.2 illustrates the effect of doping level and slab thickness on the corrective factor used for the calculation of Casimir force.

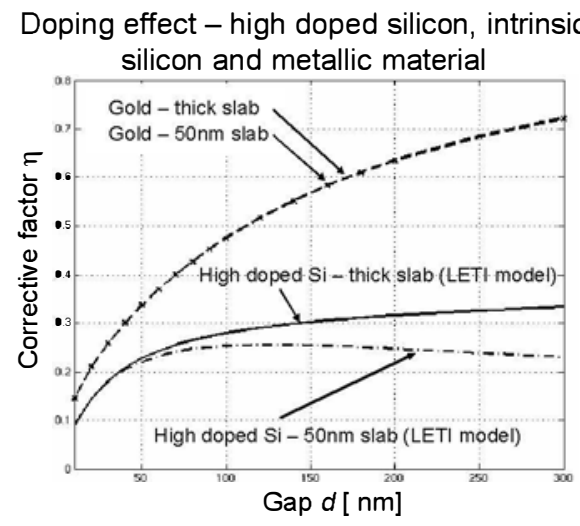

Figure 2. Effect of doping level and slab thickness on Casimir force corrective factor
When the model is used to design NEMS accelerometers, the first evident conclusion is that Casimir force can be practically neglected for gaps greater than $200 \mathrm{~nm}$ but, for smaller gaps, the impacts have quickly to be considered (Fig.3). Casimir force tends to reduce the pull-in voltage, increase the capacitance variation and reduce the linear displacement region. Fig. 3 show displacement/acceleration curves including the effect of Casimir force for IP-50G accelerometers, respectively for $130 \mathrm{~nm}$ and $400 \mathrm{~nm}$ gaps.


Figure 3. Displacement/acceleration curves for IP $50 \mathrm{G}$ accelerometer with respectively $130 \mathrm{~nm}$ and $400 \mathrm{~nm}$ gap (Influence of Casimir Force)

Fig. 4 gives expected performances for IP-50G and OOP$50 \mathrm{G}$ accelerometers.

\begin{tabular}{|c|c|c|c|}
\hline \multicolumn{2}{|l|}{ Mechanical results } & \multicolumn{2}{|l|}{ Mechanical results } \\
\hline Weigth $(\mathrm{Kg})$ & 4,10E-12 & Weigth $(\mathrm{Kg})$ & $3,64 \mathrm{E}-12$ \\
\hline Stiffness $(\mu \mathrm{N} / \mu \mathrm{m})$ & 0,07 & Stiffness $(\mu \mathrm{N} / \mu \mathrm{m})$ & 0,017 \\
\hline Mechanical sensitivity $(\mathrm{nm} / \mathrm{g})$ & 1 & Mechanical sensitivity $(\mathrm{nm} / \mathrm{g})$ & $\overline{2,6}$ \\
\hline \multicolumn{2}{|l|}{ Electrical results } & \multicolumn{2}{|l|}{ Electrical results } \\
\hline Pull-in voltage (mV) & 220 & Pull-in voltage (mV) & 110 \\
\hline Pull-in displacement (nm) & 52 & Pull-in displacement (nm) & 110 \\
\hline Co (fF) & 73 & Co (fF) & 55,3 \\
\hline C full range (50G) (fF) & 3,25 & - C full range (50G) (fF) & 28 \\
\hline Electrical sensitivity (aF/g) & 60 & Electrical sensitivity (aF/g) & 540 \\
\hline
\end{tabular}

Figure 4. Expected performances for IP $50 G$ and $00 P 50 G$ accelerometers

IP accelerometers exhibit pull-in voltage of a few $100 \mathrm{mV}$ and FS capacitance changes of a few $\mathrm{fF}$. OOP accelerometers exhibit driving voltage about a few $10 \mathrm{mV}$ 
and FS capacitance changes about a few 10fF. The sensitivities are respectively in the ranges of $100 \mathrm{aF} / \mathrm{g}$ and $500-1000 \mathrm{aF} / \mathrm{g}$ for IP and OOP accelerometers. These figures are far from conventional MEMS but can be acceptable considering the fact that electronic circuits used nowadays for high resolution accelerometers are capable to detect less than a few $10 \mathrm{aF}$ and that this kind of NEMS structures will benefit of reduced parasitic capacitances thanks to "In-IC" integration approach.

\section{THIN SOI NEMS FABRICATION}

The fabrication of NEMS accelerometers is based on Thin SOI $130 \mathrm{~nm}$ technology node (Si top layer $160 \mathrm{~nm}$, BOX $400 \mathrm{~nm}$ ) and $200 \mathrm{~mm}$ facilities. 50G, 10G and XG (higher acceleration range) were fabricated. Fig. 5 gives the simplified flow-chart used for the fabrication. The fabrication has required to address scaling considerations in order to realize on the same wafer nano-structures (springs $50 \mathrm{~nm}$, electrostatic combs $100-400 \mathrm{~nm}$ ) and large structures


reach this goal, hybrid lithography combining advanced UHR e-beam and 248nm DUV lithographies has been used. In addition, FH-vapor technique has been improved to enable the release and protection against sticking despite the $500 \mathrm{~nm}$ diameter of the release holes and the $400 \mathrm{~nm}$ thickness of the sacrificial oxide. Finally, AlSi metal was used for electrical pads.

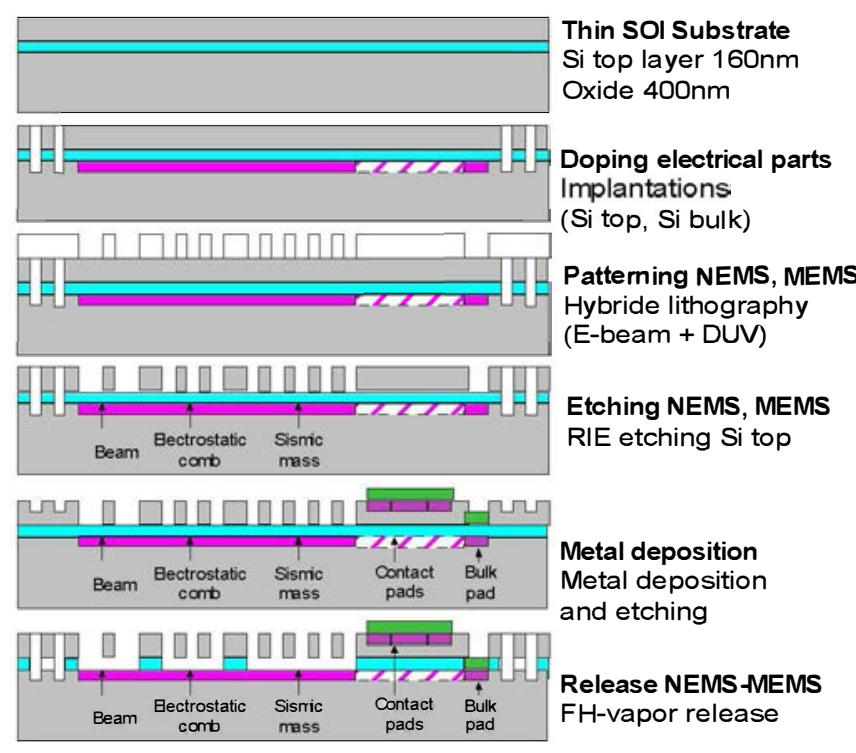

Figure 5. Simplified flow-chart for fabrication of IP \& $\bullet$ accelerometers

Fig. 6 shows how these techniques are used for the fabrication of IP accelerometers.

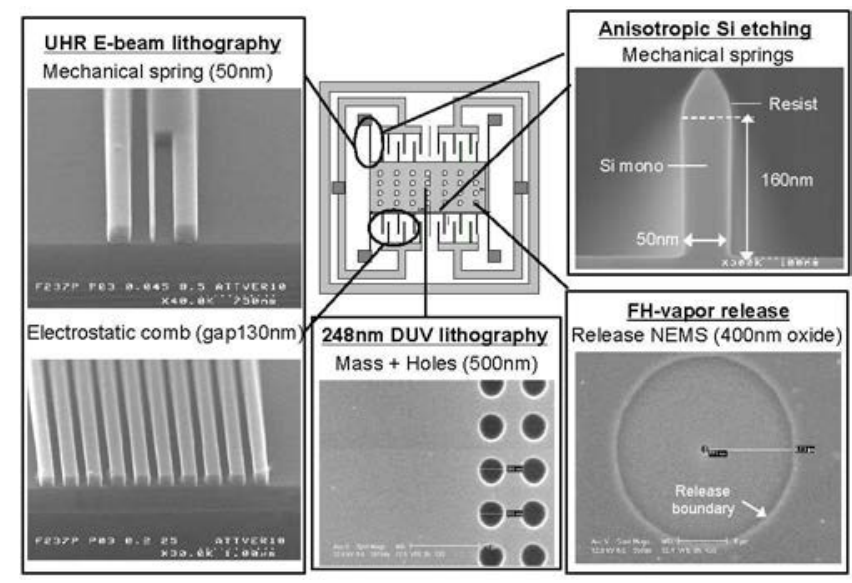

Figure 6. Technology developments for the fabrication of IP thin SOI NEMS accelerometers

Fig. 7 presents SEM photographs of fabricated thin SOI NEMS accelerometer.

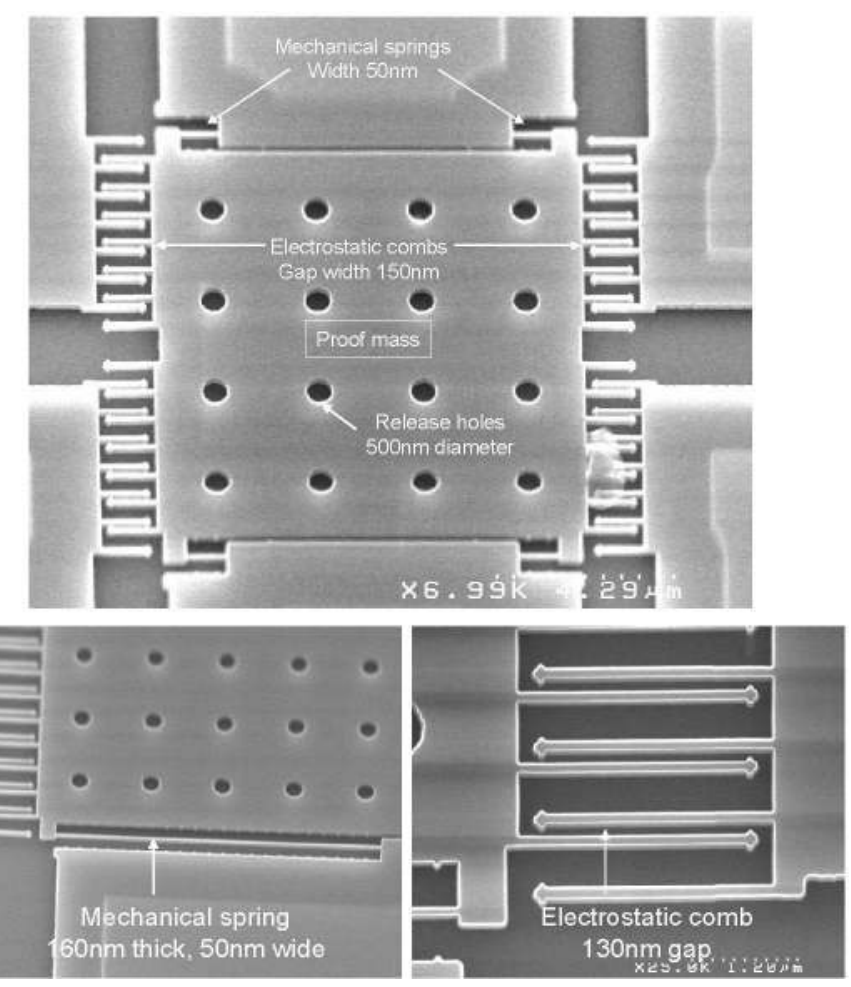

Figure 7. SEM photographs of thin SOI NEMS accelerometer (general view, detail views of mechanical spring and electrostatic comb)

The results achieved show that both nanostructures and large structures are well defined. The measured residual deflection is $<5 \mathrm{~nm}$ even with $10 \mu \mathrm{m}$ long flexible beams. Large proof masses $(75 \times 150 \mu \mathrm{m})$ are also flat, and this is also a good result if we consider the ratio of 1000 between the mass length $(150 \mu \mathrm{m})$ and the mass thickness $(160 \mathrm{~nm})$. 


\section{Characterization}

Characterization of devices including NEMS structures is really challenging because some dimensions are in the nanoscale. In addition, the structures are free standing and sometimes exhibit low stiffness. As a consequence, conventional techniques are generally limited. SEM is limited by charging effects or electron pressure and optical methods like SNOM are limited by diffraction.

To enable the characterization of NEMS, AFM technique has been investigated in order to provide measurement related to released nano-mechanical structures with typical dimensions of $160 \mathrm{~nm}$ thickness, $50 \mathrm{~nm}$ width and lateral or vertical displacements of a few $\mathrm{nm}$ to a few $10 \mathrm{~nm}$. Specific procedures have been developed in order to overcome practical hurdles encountered with free-standing NEMS, in particular to prevent breaking of free standing structures and to avoid instability occurring with low stiffness structures. Now, the technique based on AFM tapping mode is able to provide information such as substrate/lateral sticking, measurement of critical dimensions, measurement of vertical residual deflections and basic electro-mechanical behaviour, with atomic resolution for vertical axis and a few $\mathrm{nm}$ for lateral resolution.

This technique was used to investigate and characterize the behaviour of fabricated NEMS IP and OOP accelerometers : lateral dimensions such as width of mechanical spring, electrostatic teeth, electrostatic gap and also vertical deflections of released elements. Figures 8 and 9 show characterization results related to vertical residual deflections of free-standing IP and OOP accelerometers after the end of the process. It has been shown that thanks to appropriate design, some NEMS accelerometers fabricated exhibit good mechanical behaviour after process with flatness and vertical residual deflections <+/-5nm which can be considered as negligible compared to $160 \mathrm{~nm}$ thickness

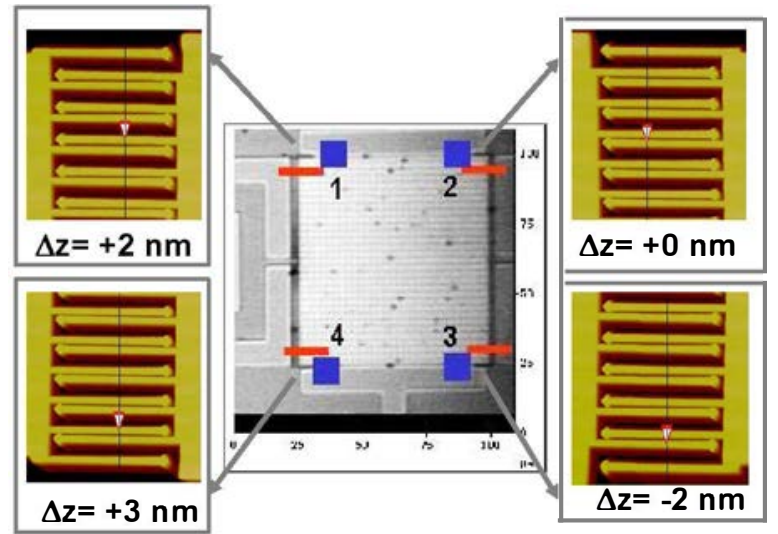

Figure 8. AFM characterization of IP $10 \mathrm{G}$ accelerometer $\Delta z$ : vertical misalignment of movable structure

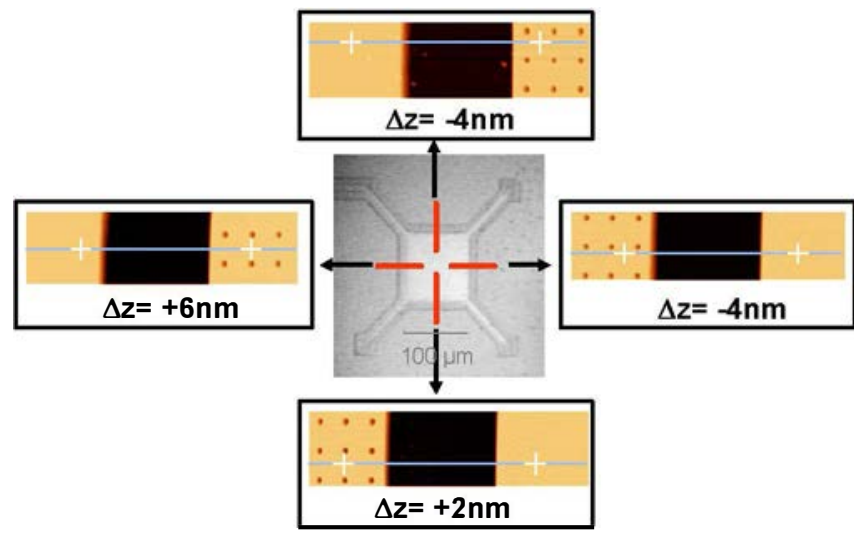

Figure 9. AFM characterization of OPP $50 \mathrm{G}$ accelerometer $\Delta z$ : vertical misalignment of movable structure

\section{Conclusion}

First Thin SOI NEMS structures for accelerometers compatible with "In-IC" integration have been designed and fabricated in a thin $160 \mathrm{~nm}$ SOI layer in order to be compatible with $0.13 \mu \mathrm{m}$ CMOS SOI technology. IP and OOP configurations have been designed and fabricated. The modeling and design take into account specific phenomenon occurring in the nano-scale, in particular Casimir force. NEMS accelerometers with mechanical springs $50 \mathrm{~nm}$ wide, electrostatic gaps 130-400nm wide have been fabricated thanks to the use of hybrid lithography combining e-beam and DUV lithography and also FH-vapor release to avoid sticking problems. In addition, AFM techniques have been investigated and optimized to characterize NEMS accelerometers. It has been shown that appropriate design and technology enable to fabricate devices exhibiting negligible residual deflections and good mechanical behaviour after process. Complementary techniques are currently under development in order to characterize electro-mechanical static and dynamic behaviour and also to investigate more in details limitations in performances.

\section{ACKNOWLEDGMENT (HEADING 5)}

Author acknowledges the support of European Commission for the funding of the integrated Project MIMOSA IST2002-507045

\section{REFERENCES}

[1] M.L. Roukes, "Nanoelectromechanical systems", Solid State Sensor and Actuator Workshop, Proceedings pp. 529-551, South Carolina, June 4-8, 2000

[2] K.L. Ekinci, Y.T. Yang, M.L. Roukes, "Ultimate limits to inertial mass sensing based upon nanoelectromechanical systems", Journal of Applied Physics, V95, N•5, pp. 2685-2689, March 2004

[3] J.D. Aldridge et al, "Nanoelectronic and nanomechanical systems", SPIE Proceedins 4591, 11, 2001

[4] http://www.mimosa-fp6.com 